\title{
Urban Computing: Key Challenges and Issues of Traffic Management System
}

\author{
Mamta \\ Asst. Prof. \\ Ymcaust Faridabad
}

\author{
C. K. Nagpal \\ Professor \\ Ymcaust Faridabad
}

\begin{abstract}
Urban Computing collects heterogeneous data from diverse sources,integrates it and analyzes it to deal with the challenges faced by the Urban cities. Due to rapid urbanization it has emerged as an important research area. Urban computing connects unobtrusive and ubiquitous sensing technologies, advanced data management and analytics models, and novel visualization methods, to create win-win-win solutions that improve urban environment, human life quality, and city operation systems. It has various areas of application include cultural archiving, energy consumption, health, social interaction, transportation and environment. In this paper we discuss various key challenges and issues related to traffic management in urban cities.
\end{abstract}

\section{Keywords}

Urban computing, urban informatics, big data, human mobility, urban sensing, knowledge fusion, computing with heterogeneous data.

\section{INTRODUCTION}

Due to the rapid progress of urbanization many people's lives have been modernized, but it has led to many big challenges, such as air pollution, increased energy consumption, and traffic congestion. Earlier it was nearly impossible to tackle these challenges due to the complex and dynamic design of cities. Nowadays, variety of big data in urban spaces is available that is collected by sensing technologies and largescale computing infrastructures obtained from human mobility, air quality, traffic patterns, and geographical data. The big data i.e. collected from the vast sources can help us to tackle these challenges when used correctly. For instance, to detect a city's road network anomaly due to traffic by analyzing the city-wide human mobility data. The pollution level in a city can also be formulated by analyzing the traffic flow. With the motivation of building more intelligent cities, the vision of urban computing helps to unlock the power of knowledge from big and heterogeneous data collected in urban space.

Urban computing is a key research idea from a long time but still it has many questions.

a) What are the research problems that can be solved using urban computing?

b) How this system works?

c) What are its domain areas?

To address these issues, urban computing is defined then its framework, key challenges, data available for traffic management and then its usage in solving traffic related problem with the use of some technique referred from [1]Urban Computing: Concepts, Methodologies, and Applications by YU ZHENG et al. Urban computing is a multidisciplinary research field, where computer science meets conventional city-related areas, such as civil engineering, transportation, economics, energy engineering, environmental sciences, ecology, and sociology. This paper mainly focuses on the urban computing and discusses a traffic related issues in urban computing.

\section{FRAMEWORK OF URBAN COMPUTING}

Urban computing is a process of collecting big and heterogeneous data from diversified sources such as sensors, vehicle, building, devices, human generated data and the integrating and analyzing the data to tackle the issues that a city faces. Urban computing connects unobtrusive and ubiquitous sensing technologies, advanced data management and analytic models, and novel visualization methods to create win-win-win solutions that improve urban environment, human life quality, and city operation systems. Urban computing deals with many traditional fields like transportation, civil engineering, economy, ecology, and sociology in the context of urban spaces.

As shown in Fig. 1 the data from diverse sources (human dynamics), which is called as Big data, is collected by the prosumers, a proper understanding is done by implementing this data on urban computing modals and then a feedback is given which help to take decision for an issue.

General Framework:

The general framework as shown in Fig. 2 consists of four layers: urban sensing, urban data management, data analytics, and service providing.

As shown in figure 2 in urban sensing and data acquisition step the data from various source such as traffic data, black box data, air quality, road network data, human mobility data is acquired using various methods such as participatory sensing, mobile sensing. In data management step a structure is provided to this data using some techniques such as trajectory formation etc. then data mining and visualization is done on this data to form a pattern and to understand this data which ultimately helps to provide many services such as easing traffic, to control air pollution etc.

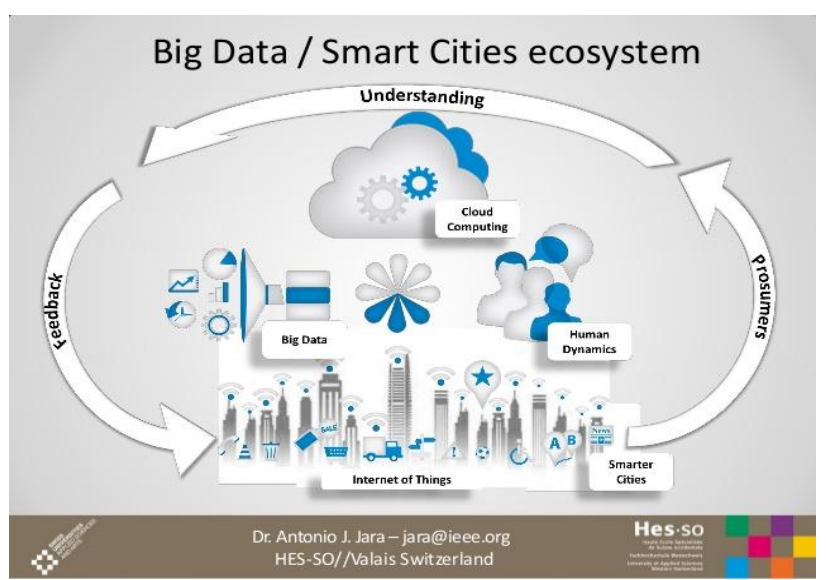

Fig. 1: Use of Big data in urban computing to develop a smart city 
Using urban anomaly detection as an example the operation of the framework is as follows:

In the urban sensing and data acquisition step, using GPS sensors as mentioned in [3] by s.castro Et.al.2013 and the mobile phone signals, constant probing of people's mobility as mentioned in [5] C. Gonz'alez et. al 2008" Understanding individual human mobility patterns". can be done and the social media posted by people on internet is also collected. In the data management step, the human mobility and social media data are well organized by some indexing structure that incorporates spatiotemporal information and texts for supporting efficient data analytics. In the data analytics step, using the previous information, the locations are identified where people's mobility significantly differs from its origin patterns. In the meantime, the location of the anomaly can also be known by mining terms from the social media that are related to the locations and time span. In the service providing step, the location information is sent to nearby drivers so that they can choose a bypass. In addition, the information can be delivered to the transportation authority for dispersing traffic and diagnosing the anomaly. The system can be continued to detect urban anomalies, which in turn improve people's driving experiences and reduce traffic congestion taken from.

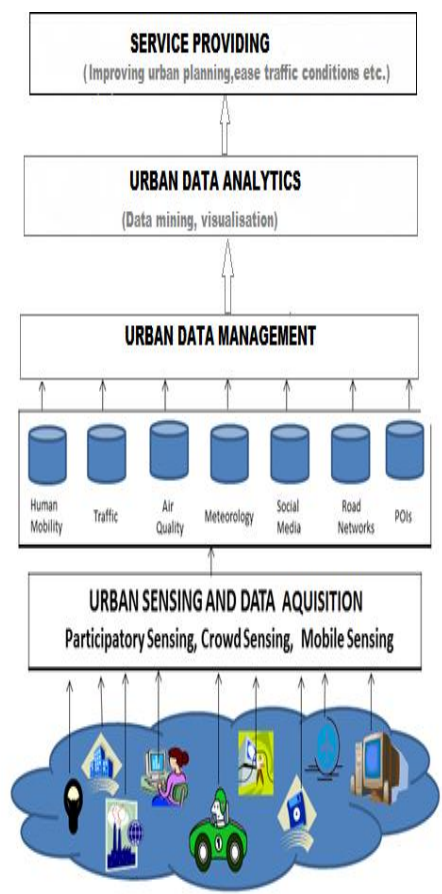

Fig. 2: Urban computing framework

Compared with other systems (e.g., web search engines) that are based on a single (modal)-data/single-task framework (i.e., information retrieval from web pages), urban computig holds a multi (modal)-data/multitask framework. The tasks of urban computing include improving urban planning, easing traffic congestion, reducing energy consumption, and reducing air pollution however the focus is mainly on easing traffic congestion.

\section{KEY CHALLENGES}

To achieves the goals of urban computing using this framework there are many key challenges in each step discussed below:

\subsection{Urban Sensing and Data Acquisition}

The data acquisition technique continually collects data on a citywide basis. Sensing can be done by monitoring traffic flow on the road but continually probing is not feasible as enough sensors are not available to us and installing that many sensors will be very much costly. Humans as a sensor is a new concept that may help tackle this challenge. The data posted on social media help us to understand the events happening around the people. The GPS sensors in the vehicle of people who are driving on the .1road help us to know the traffic condition in that area. However, despite the flexibility it brings a few problems:

- Energy consumption and privacy: when users proactively contribute their data (usually using a Smartphone) to save the energy of a Smartphone and protect their privacy during the sensing process. There is a tradeoff among energy, privacy, and the utility of shared data [12] [Xue et al. 2013].

- Loose-controlled and non uniform distributed sensors: Sensors are used to sense the data and configured to send reading at a certain frequency but people send their data at any time and sometimes they may not send any data. At some moments there may be a place where there is no person and we do not have any sensor which creates data sparsity problem.

- Unstructured, implicit, and noise data: The data generated by traditional sensors is well structured, explicit, clean, and easy to understand. However, the data contributed by users is usually in a free format, such as texts and images, or cannot explicitly lead us to the final goal as when using traditional sensors. Sometimes, the information from human sensors is also quite noisy.

\subsection{Computing with Heterogeneous Data}

- Learn mutually reinforced knowledge from heterogeneous data: Existing data-mining and machine-learning techniques usually handle one kind of data out of broad range of factors (e.g., exploring air pollutions involves the simultaneous study of traffic flow, meteorology, and land uses) for example, computer vision deals with images, and natural language processing is based on texts. According to studies [Zheng et al. 2013b; Yuan et al. 2012], equally treating the features extracted from different data sources by putting these features into a feature vector and throwing them into a classification model does not achieve the best performance. So there is a requirement to generate advance data analytics models that can learn mutually reinforced knowledge among multiple heterogeneous data generated from different sources, including sensors, people, vehicles, and buildings.

- Both effective and efficient learning ability: Many urban computing scenarios (e.g., detecting traffic anomalies and monitoring air quality) require instant answers. Besides just increasing the number of machines to speed up the computation, aggregate data management, mining and machine-learning algorithms into a computing framework to provide both effective and efficient knowledge discovery ability is required. In addition, traditional data 
management techniques are usually designed for a single modal data source. An advanced management methodology that can organize multimodal data (such as streaming, geospatial, and textual data) well is still missing. So, computing with multiple heterogeneous data is a fusion of data and algorithms.

- Visualization: Big data provides us a lot of information so it needs to be presented better. A good visualization of original data could inspire new ideas to solve a problem, while the visualization of computing results can reveal knowledge intuitively so as to help in decision making. The visualization of data may also suggest the correlation or causality between different factors. The multimodal data in urban computing scenarios leads to high dimensions of views, such as spatial, temporal, and social, for a visualization. How to interrelate different kind of data in different views and detect patterns and trends is challenging.

\subsection{Hybrid Systems Blending the Physical and Virtual Worlds}

Here the physical world data obtained from sensors and virtual world that obtained from social media sites is integrated. The data is then sent to cloud systems. After the data is processed with other data sources in the cloud, the knowledge learned from the data is used to serve users from the physical world via mobile clients (e.g., driving direction suggestions, taxi ridesharing, and air quality monitoring).since the systems needs to communicate with many devices and users simultaneously and send and receive data of different formats and at different frequencies, accomplishing this task was much more challenging.

\section{DATA AVAILABLE FOR TRAFFIC MANAGEMENT}

Traffic data: Traffic data can be collected in many ways such as using loop sensors, surveillance cameras, and floating cars. Loop sensors are usually embedded in pairs in major roads e.g. highways. These sensors detect the time interval travelled by a particular vehicle between a pair of detector. On the basis of calculated distance between a pair of loop detectors, calculate the speed of the vehicle. Counting the number of vehicles and traversing a pair of loop detectors in a time slot, the traffic volume on a road can be known. As deploying and maintaining loop sensors is very expensive in terms of money and human resources, such traffic monitoring technology is usually employed for major roads rather than low-level streets. As a result, the coverage of loop sensors is quite limited. Secondly many Surveillance cameras can be widely deployed in urban areas, generating a huge volume of images and videos reflecting traffic patterns. The data provides the actual traffic conditions to people. However, it is still a challenging task to automatically turn the images and videos into a specific traffic volume and travel speed. Hence monitoring citywide traffic conditions through this approach is mainly based on human effort. Lastly,[7] X. Pang et al. explains floating car data that can be generated by vehicles traveling around a city with a GPS sensor. The trajectories of these vehicles will be sent to a central system and matched to a road network for deriving speeds on road segments. As many cities have already installed GPS sensors in taxicabs, buses, and logistics trucks for different purposes, floating car data has already been widely available. In contrast to loop sensors and surveillance camera-based approaches, floating car-based traffic monitoring methods have higher flexibility and a lower deployment cost. However, the coverage of floating car data depends on the distribution of the probing vehicles, which may change over time and be skewed in a city in a time span. In other words, the data sparsity problem still exists, calling for advanced knowledge discovery technology that can recover the citywide traffic conditions based on limited data. Castro et al. [2013] presents a survey on turning GPS trajectories of taxis into social and community dynamics.

\section{URBAN COMPUTING FOR TRANSPORTATION SYSTEMS 5.1 Improving Driving Experiences}

With the help of urban computing we are able to find fast driving routes which saves both the time of the driver and energy consumption. Intensive studies have been done to learn historical traffic patterns estimate real-time traffic flows and forecast future traffic conditions on individual road segments in terms of floating car data, such as GPS trajectories of vehicles, Wi-Fi, and GSM signals. However, work modeling the citywide traffic patterns is still rare.

VTrack [Thiagarajan et al. 2009] is a system for travel time estimation based on Wi-Fi signals, measuring and localizing the time delays. The system uses a hidden Markov model (HMM)-based map matching scheme that interpolates sparse data to identify the most probable road segments driven by the user. A travel time estimation method is then proposed to attribute travel times to those segments. The experiments show that VTrack can tolerate significant noise and outages in these location estimates and still successfully identify delayprone segments.

T-Drive [Yuan and Zheng et al. 2010a, 2011b, 2013b] is a system that provides personalized driving directions that adapt to weather, traffic conditions, and a person's own driving habits. The first version of this system only suggests the practically fastest path based on historical trajectories of taxicabs. The key insights consist of two parts: (1) GPSequipped taxicabs can be regarded as mobile sensors continually probing the traffic patterns on road surfaces, and (2) taxi drivers are experienced drivers who can find a really quick route based on their knowledge, which incorporates not only the distance of a route but also the traffic conditions and the probability of accidents. So, the taxi trajectories imply traffic patterns and human intelligence.

\subsection{Improving Taxi Services}

Taxis are the important services that help to commute many people to their place. Effectively connecting passengers with vacant taxis is of great importance to saving people's waiting time, increasing taxi drivers' profit, and reducing unnecessary traffic and energy consumption. To address this issue, three categories of research have been done:

\subsection{Taxi dispatching Systems}

These systems[Lee et al. 2004] assign the taxi to user on the basis of user request. These systems also allow a user to book a taxi in advance, therefore reducing the flexibility of taxi services. Some real- time dispatching systems provide the services based on the nearest neighbor principle of distance and time. The main challenge the system is faced with is the uncertainty of taxis' movement when searching for taxis.

\subsection{Taxi Recommendation Systems}

This category of systems approaches the problem from the perspective of recommendation. Ge et al.[2010] developed a 
mobile recommender system, which has the ability to recommend a sequence of pick-up points for taxi drivers or a sequence of potential parking positions. The goal of the system is to maximize the probability of business success and reduce energy consumption. T-Finder [Yuan and Zheng et al. 2011a, 2014] provides taxi drivers with some locations and the routes to these locations, toward which they are more likely to pick up passengers quickly (during the routes or in these locations) and maximize the profit of the next trip.

\subsection{Taxi Ridesharing Services}

Taxi ridesharing is of great importance to save energy consumption and easing traffic congestion while satisfying people's need to commute. T-Share [Ma and Zheng et al. 2013] is a large-scale dynamic taxi-sharing system that accepts passengers' real-time ride requests sent from Smartphone and schedules taxis to pick up passengers via ridesharing, subject to time, capacity, and monetary constraints.

Except this the public transportation system can be improved by improving bus services, subway services and bike sharing system.

\section{CONCLUSION}

To achieve high performance traffic management using urban computing many other techniques can be used such as making trajectory for the different locations. In this paper the main discussion is about the general framework of urban computing which can help to mange many key challenges such as environment pollution, traffic, road networks etc. has been discussed with a focus on traffic data and its use to sort out many traffic related problems.

\section{REFERENCES}

[1] Urban Computing: Concepts, Methodologies, and Applications by YU ZHENG,(Microsoft Research), LICIA CAPRA, (University College London), OURI WOLFSON, (University of Illinois at Chicago), HAI YANG, (Hong Kong University of Science and Technology).

[2] www.slideshare.net/Mining In The Middle Of The City: The need of Big Data for Smart city,

[3] S. Castro, D. Zhang, C. Chen, S. Li, and G. Pan. 2013. From taxi GPS traces to social and community dynamics: A survey. ACM Computer Survey 46, 2, Article 17, 34 pages

[4] Y. Zheng, and J. Hu. 2012. Inferring the root cause in road traffic anomalies. In Proceedings of the 2012 IEEE International Conference on Data Mining. IEEE, 141150 .
[5] C. Gonz'alez, C. A. Hidalgo, and A. L. Barab’asi. 2008. Understanding individual human mobility patterns. Nature 453, 7196, 779-782.

[6] Y. Zheng,and O.Wolfson.2013. T-Share: A large-scale dynamic taxi ridesharing service. In Proceedings of IEEE International Conference on Data Engineering IEEE.

[7] X. Pang, S. Chawla, W. Liu, and Y. Zheng. 2013. On Detection of Emerging Anomalous Traffic Patterns Using GPS Data. Data and Knowledge Engineering (DKE) 87, 357-373.

[8] J. Yuan, Y. Zheng, X. Xie, and G. Sun. 2011b. Driving with knowledge from the physical world. In Proceedings of 17th SIGKDD Conference on Knowledge Discovery and Data Mining. ACM, 316-324

[9] J. Yuan, Y. Zheng, C. Zhang, W. Xie, X. Xie, G. Sun, and Y. Huang. 2010a. T-Drive: Driving directions based on taxi trajectories. In Proceedings of ACM SIGSPATIAL Conference on Advances in Geographical Information Systems. ACM, 99-108. J. Yuan, Y. Zheng, C. Zhang, X. Xie, and G. Sun. 2010

[10] J. Yuan, Y. Zheng, X. Xie, and G. Sun. 2013b. T-Drive: Enhancing driving directions with taxi drivers' intelligence. Transactions on Knowledge and Data Engineering 25, 1, 220.

[11] Y. Zheng, Y. Chen, Q. Li, X. Xie, and W. Y. Ma. 2010b. Understanding transportation modes based on GPS data for web applications. ACM Transactions on the Web 4, $1,1-36$.

[12] Y. Xue, R. Zhang, Y. Zheng, X. Xie, J. Huang, and Z. $\mathrm{Xu}$. 2013. Destination prediction by sub-trajectory synthesis and privacy protection against such prediction. In Proceedings of the 29th IEEE International Conference on Data Engineering. IEEE, 254-265.

[13] Thiagarajan, L. Ravindranath, K. LaCurts, S. Madden, H. Balakrishnan, S. Toledo, and J. Eriksson. 2009. VTrack: Accurate, energy-aware road traffic delay estimation using mobile phones. In Proceedings of the 7th ACM Conference on Embedded Networked Sensor Systems.

[14] Lee, H. Wang, R. Cheu, and S. Teo. 2004. Taxi dispatch system based on current demands and real- time traffic conditions. Transportation Research Record: Journal of the Transportation Research Board, 1882(-1):193-200.

[15] Yuan, Y. Zheng, L. Zhang, X. Xie, and G. Sun. 2011a. Where to find my next passenger? In Proceedings of 13th ACM International Conference on Ubiquitous Computing. ACM, 109-118. 